\title{
Innermost stable circular orbits of neutron stars in dilatonic-Einstein-Gauss-Bonnet theory
}

\author{
Guillermo A. González ${ }^{1}$, Burkhard Kleihaus ${ }^{2}$, Jutta Kunz ${ }^{2}$ and Sindy Mojica ${ }^{1}$ \\ ${ }^{1}$ Grupo de Investigación en Relatividad y Gravitación, Escuela de Física, \\ Universidad Industrial de Santander, A. A. 678, Bucaramanga 680002, Colombia. \\ ${ }^{2}$ Institut für Physik, Universität Oldenburg, D-26111 Oldenburg, Germany
}

(Dated: December 7, 2018)

\begin{abstract}
The innermost stable circular orbits (ISCOs) around rapidly rotating neutron stars are studied in dilatonic Einstein-Gauss-Bonnet theory. Universal relations for properly scaled ISCO properties are extended from General Relativity to dilatonic Einstein-Gauss-Bonnet theory and additional relations are obtained.
\end{abstract}

PACS numbers:

\section{INTRODUCTION}

Currently General Relativity (GR) fully describes the behaviour of the gravitational force, as tested by experiments and observations in the solar system and beyond, involving both weak and strong gravitational fields [1 $[3]$. However, in the quest of a better understanding of all fundamental forces of nature, gravity is still the only one without an appropriate description at the quantum level. Thus, although GR is continuing to pass all tests, there are various attempts to formulate a quantum theory of gravity.

Here one of the popular contenders is String Theory, which in its low energy limit has led to effective field theories, whose predictions can be tested analogously to those of GR [1 3$]$. String Theory predictions include the presence of higher curvature terms and additional fields [4 [6]. Among the String Theory motivated alternative theories of gravity dilatonic Einstein-Gauss-Bonnet theory (dEGB) has led to particular interest because it yields only second order equations of motion, and thus it is ghost free.

Astrophysical compact objects are an important probe to test the range of validity of GR and alternative theories of gravity like dEGB, because their strong gravitational fields may provide information on how gravity behaves under extreme conditions. Therefore deviations from the predictions of GR may arise due to the presence of higher curvature terms and additional fields. In dEGB theory the higher curvature terms enter via the Gauss-Bonnet (GB) term, that is coupled to a scalar dilaton field, see e.g. 7]. EGBd theory may also be considered as a particular case of Horndeski gravity [8].

Black hole solutions have been studied in dEGB theory for quite some time [9 20] starting with the perturbative solutions, and then extending them to fully nonperturbative static and rotating black hole solutions. Interesting features of these dEGB black holes include a minimal mass for fixed coupling constants, and a violation of the Kerr limit, i.e., angular momenta exceeding $J / M^{2}=1$.

Neutron stars, on the other hand, are not as simple objects as black holes, since the interior solutions of the Einstein field equations must be taken into account, as well. This means that the stress-energy tensor contributes decisively, when obtaining the solutions of the Einstein equations, and an equation of state (EOS) describing the interior structure must be included. However, the EOS of matter under such extreme conditions as found inside a neutron star is yet unknown [21].

This current uncertainty in the EOS is mainly due to the limitations of Earth based laboratories to achieve the huge densities present in neutron star cores. Indeed, there exists a large body of literature on the neutron star EOS [21 23]. These EOS range from purely hadronic matter to hybrid quark-hadron matter, all the way to pure quark matter, and thus giving rise to quark stars. The choice of the EOS employed then dictates the physical properties of these compact stars, such as their radii and masses. Observations of neutron star masses on the order of $M \approx 2 M_{\odot}[24,25]$ thus provide a strong selection criterion for viable EOS.

A valuable tool to further our understanding of the properties of neutrons stars are their so-called universal relations. Such universal relations represent intriguing relations between appropriately scaled physical properties of neutron stars, which are almost independent of the EOS (see e.g. the recent reviews [26, 27]). In these universal relations compactness plays an important role. Most prominent among these relations are the $I$-Love$Q$ relations of neutron stars between their moments of inertia $I$, their Love numbers, and their quadrupole moments $Q[28,34]$.

While neutron stars and their properties have been widely studied in GR, also in the rapidly rotating case 35 41], much less is known about neutron stars in alternative theories of gravity. A recent review on the state of 
the art can be found in [3]. In dEGB theory neutron stars have been investigated in [42 [45], including both the static and the rapidly rotating case, with associated $I-Q$ relations, yielding little deviation from the well-known GR case (similarly to the case of scalar-tensor theory [46]).

In this paper, rotating neutron stars in dEGB are considered for two EOS referred to as DI-II [47] and FPS [48, 49], for which the $2 M_{\odot}$ limit [24, 25] is only reached and exceeded for fast rotating neutron stars [44, 45]. Various properties of these dEGB neutron stars have been discussed before [42 45]. In particular, it has been shown, that with increasing GB coupling constant the maximum mass of the neutron stars decreases.

However, an analysis for test particles surrounding a rotating dEBG neutron star has not been performed so far, although such an analysis has been made for black holes in dEGB (see [17,20]). It is therefore a natural important next step to investigate the innermost stable circular orbit (ISCO) [50, 51] for dEGB neutron stars

following a similar procedure. Despite the numerical limitations, this may in fact reveal significant effects of the scalar field on test particles orbiting around a rotating dEBG neutron star.

In GR the ISCO has been studied for numerous rotating neutron star models and EOSs (see e.g., 52 631). Concerning alternative theories of gravity the ISCO for rotating neutron stars has been addressed in [42, 64, 65]. Important astrophysical applications of the ISCO concern the description and understanding of accretion disks around neutron stars. Moreover, it has been suggested that the ISCO has a direct relation with quasi-periodic oscillations (QPOs). This phenomenon occurs in x-ray binaries and is considered a relevant neutron star signature. In fact, the ISCO may be interpreted as the upper bound of the observed frequency provided by x-ray observations [66, 67].

In section II we present the theoretical setting for our investigations, including the dEGB action, the metric, the EOS, and the equations of motion for the ISCOs. We discuss our results in section III, starting with a brief recollection of the domain of existence of the dEGB neutron stars. We then present the ISCOs for these neutron star models, and address their universal relations. We present our conclusions in section IV.

\section{THEORETICAL SETTING}

\section{A. dEGB gravity}

The action describing dilatonic-Einstein-Gauss-Bonnet gravity is given by

$$
S=\frac{1}{16 \pi G} \int d^{4} x \sqrt{-g}\left[R-\frac{1}{2}\left(\partial_{\mu} \phi\right)^{2}+\alpha e^{-\gamma \phi} R_{\mathrm{GB}}^{2}\right]+S_{\text {matter }}
$$

where $G$ is Newton's constant, $R$ is the curvature scalar, $\phi$ denotes the dilaton field, $\alpha$ and $\gamma$ are coupling constants, $R_{G B}^{2}$ denotes the Gauss-Bonnet term,

$$
R_{G B}^{2}=R_{\mu \nu \rho \sigma} R^{\mu \nu \rho \sigma}-4 R_{\mu \nu} R^{\mu \nu}+R^{2},
$$

while the action for the nuclear matter is symbolized by $S_{\text {matter }}$.

The gravitational field equations are then given by

$$
\begin{aligned}
G_{\mu \nu}= & \frac{1}{2}\left[\nabla_{\mu} \phi \nabla_{\nu} \phi-\frac{1}{2} g_{\mu \nu} \nabla_{\lambda} \phi \nabla^{\lambda} \phi\right] \\
& -\alpha e^{-\gamma \phi}\left[H_{\mu \nu}+4\left(\gamma^{2} \nabla^{\rho} \phi \nabla^{\sigma} \phi-\gamma \nabla^{\rho} \nabla^{\sigma} \phi\right) P_{\mu \rho \nu \sigma}\right] \\
& +8 \pi G T_{\mu \nu},
\end{aligned}
$$

where

$$
\begin{gathered}
H_{\mu \nu}=2\left[R R_{\mu \nu}-2 R_{\mu \rho} R_{\nu}^{\rho}-2 R_{\mu \rho \nu \sigma} R^{\rho \sigma}+R_{\mu \rho \sigma \lambda} R_{\nu}^{\rho \sigma \lambda}\right]-\frac{1}{2} g_{\mu \nu} R_{\mathrm{GB}}^{2}, \\
P_{\mu \nu \rho \sigma}=R_{\mu \nu \rho \sigma}+2 g_{\mu[\sigma} R_{\rho] \nu}+2 g_{\nu[\rho} R_{\sigma] \mu}+R g_{\mu[\rho} g_{\sigma] \nu} .
\end{gathered}
$$

Note that in four dimensions $H_{\mu \nu}=0$.

The stress-energy tensor $T_{\mu \nu}$ of the nuclear matter occurring on the right hand side of the field equations is taken to be in the form of a perfect fluid

$$
T_{\mu \nu}=(\varepsilon+P) U_{\mu} U_{\nu}+P g_{\mu \nu}
$$


with energy density $\varepsilon$, pressure $P$, and four velocity $U_{\mu}$ of the fluid. In hydrostatic equilibrium the stress-energy tensor is covariantly conserved,

$$
\nabla_{\mu} T^{\mu \nu}=0
$$

Finally, the dilaton field equation is given by

$$
\nabla^{2} \phi=\gamma \alpha e^{-\gamma \phi} R_{\mathrm{GB}}^{2} .
$$

For the GB coupling constant $\alpha$ three values are chosen, $\alpha=0$ (GR limit), $\alpha=1$ and $\alpha=2$, while for the dilaton coupling constant $\gamma$ the string value $\gamma=1$ is fixed (see our previous study for details [45], where also observational restrictions for the coupling parameter $\alpha$ are discussed, following [42, 68] ).

\section{B. Metric and fluid}

The metric describing the stationary, axially symmetric spacetime is specified in terms of the spherical coordinates $r$ and $\theta$, and reads in quasi-isotropic metric [69]

$$
d s^{2}=g_{\mu \nu} d x^{\mu} d x^{\nu}=-e^{2 \nu_{0}} d t^{2}+e^{2\left(\nu_{1}-\nu_{0}\right)}\left(e^{2 \nu_{2}}\left[d r^{2}+r^{2} d \theta^{2}\right]+r^{2} \sin ^{2} \theta(d \varphi-\omega d t)^{2}\right)
$$

with metric functions $\nu_{0}(r, \theta), \nu_{1}(r, \theta), \nu_{2}(r, \theta)$ and $\omega(r, \theta)$.

For a uniformly rotating neutron star fluid the four velocity has the form

$$
U^{\mu}=(u, 0,0, \Omega u)
$$

where the constant angular velocity of the fluid is denoted by $\Omega$. The normalization condition for the four velocity of the fluid $U^{\mu} U_{\mu}=-1$ can then be utilized to express the velocity function $u$ in terms of the metric functions $\nu_{0}, \nu_{1}$ and $\omega$ and the fluid angular velocity $\Omega$,

$$
u^{2}=\frac{e^{-2 \nu_{0}}}{1-(\Omega-\omega)^{2} r^{2} \sin ^{2} \theta e^{2 \nu_{1}-4 \nu_{0}}} .
$$

The set of equations (6), $\nabla_{\mu} T^{\mu \nu}=0$, result in the differential equations for the pressure $P$

$$
\frac{\partial_{r} P}{\varepsilon+P}=\frac{\partial_{r} u}{u}, \quad \frac{\partial_{\theta} P}{\varepsilon+P}=\frac{\partial_{\theta} u}{u},
$$

and need to be supplemented by an EOS, $\varepsilon=\varepsilon(P)$, relating the energy density $\epsilon$ to the pressure.

In a polytropic EOS the pressure $P$ is related directly to the baryon mass density $\rho$ via [40]

$$
P=K \rho^{\Gamma}, \quad \Gamma=1+\frac{1}{N}
$$

where $K$ is the polytropic constant $K, \Gamma$ the polytropic exponent $\Gamma$, and $N$ the polytropic index $N$. The energy density $\varepsilon$ of the fluid is then given by

$$
\varepsilon=N P+\rho .
$$

Based on the calculations presented in [45], we here have taken two EOS, the polytropic DI-II EOS [47] with polytropic index $N=0.7463$ and polytropic number $K=1189.0$, and the FPS EOS [48, 49] with polytropic parameters $N=0.6104$ and $K=5392.0$.

\section{Innermost stable circular orbits}

The stability of circular orbits is physically relevant for studies of accretion disks of particles orbiting around compact objects such as black holes or neutron stars. Instabilities due to perturbations of the circular orbits in 
the equatorial plane and perpendicular perturbations are decoupled. In this paper our interest is focused only on the first case [51].

The general set of geodesics is obtained from the Lagrangian

$$
2 \mathcal{L}=e^{-2 \beta \phi} g_{\mu \nu} \dot{x}^{\mu} \dot{x}^{\nu}
$$

together with the normalization condition

$$
e^{-2 \beta \phi} g_{\mu \nu} \dot{x}^{\mu} \dot{x}^{\nu}=-\epsilon,
$$

where $\epsilon=0$ and 1 for massless and massive particles, respectively, and the coupling between matter and the dilaton field is associated with the coupling constant $\beta$, which in heterotic string theory assumes the value $\beta=0.5$ [15]. The derivative with respect to the affine parameter along the geodesics is denoted by a dot.

Explicitly we find for massive particles orbiting in the equatorial plane $(\theta=\pi / 2)$ the Lagrangian $\mathcal{L}$, resp. normalization condition

$$
\begin{aligned}
2 \mathcal{L} & =e^{-2 \beta \phi}\left[\left(-e^{2 \nu_{0}}+\omega^{2} r^{2} e^{2\left(\nu_{1}-\nu_{0}\right)}\right) \dot{t}^{2}+e^{2\left(\nu_{1}-\nu_{0}+\nu_{2}\right)} \dot{r}^{2}+e^{2\left(\nu_{1}-\nu_{0}\right)} r^{2} \dot{\varphi}^{2}-2 \omega e^{2\left(\nu_{1}-\nu_{0}\right)} r^{2} \dot{t} \dot{\varphi}\right] \\
-1 & =e^{-2 \beta \phi} g_{\mu \nu} \dot{x}^{\mu} \dot{x}^{\nu}
\end{aligned}
$$

Stationarity and axial symmetry imply the existence of two Killing vectors associated with two conserved quantities, the particle energy $E$ and angular momentum $L$,

$$
\begin{aligned}
& E=-\frac{\partial \mathcal{L}}{\partial \dot{t}} \\
& L=\frac{\partial \mathcal{L}}{\partial \dot{\varphi}}
\end{aligned}
$$

The full set of equations of motion for a massive particle orbiting in the equatorial plane then becomes

$$
\begin{gathered}
\dot{t}=(E-\omega L) e^{-2 \nu_{0}} e^{2 \beta \phi} \\
\dot{\varphi}=\left(\omega(E-\omega L) e^{-2 \nu_{0}}+\frac{L}{r^{2}} e^{2\left(\nu_{0}-\nu_{1}\right)}\right) e^{2 \beta \phi} \\
\dot{r}^{2}=\frac{e^{4 \beta \phi}}{e^{2\left(\nu_{1}+\nu_{2}\right)}}\left(E-V_{+}\right)\left(E-V_{-}\right) \equiv V(r), \text { with } V_{ \pm}=\omega L+\sqrt{e^{2 \nu_{1}} e^{-2 \beta \phi}+\frac{L^{2}}{r^{2}} e^{2 \nu_{0}}} e^{\nu_{0}-\nu_{1}},
\end{gathered}
$$

where for the last equation the normalization condition Eq. (17) has been used.

The orbital angular velocity of the particle is obtained via

$$
\Omega_{c}=\frac{\dot{\varphi}}{\dot{t}}=\omega+\frac{1}{r^{2}} \frac{L e^{4 \nu_{0}-2 \nu_{1}}}{E-L \omega} .
$$

We note that for circular orbits the potential satisfies $V(r)=V^{\prime}(r)=0$. These conditions yield two relations for the particle energy $E$ and angular momentum $L$ to be solved. The resulting two sets of solutions correspond to corotating and counterrotating orbits.

In order to obtain stable orbits, the second derivative of the effective potential should be required to be negative, $V^{\prime \prime}(r)<0$. At a particular value of the radial coordinate $r=r_{\text {ISCO }}$ the stability changes, with the stable circular orbits residing at $r>r_{\mathrm{ISCO}}$.

\section{RESULTS}

In the following we first recall the properties of the dEGB and GR neutron star models. Then we discuss the ISCO radii and frequencies for these models. Subsequently we address universal relations for the ISCOs in GR and dEGB theory.

In the following we will adapt units such that $\Omega$ and $\Omega_{c}$ are in units of $c / R_{0}=2.031 \times 10^{5} s$, unless otherwise stated. 


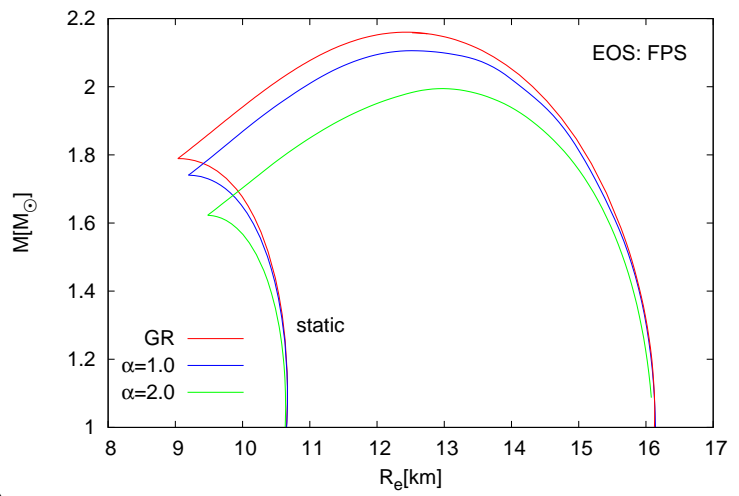

FIG. 1: (a) Mass-radius relation for the physically relevant domain of neutron star models, limited by the static solutions, the solutions at the Kepler limit and the solutions at the secular instability line for GB coupling constants $\alpha=0,1$ and 2. (a) EOS FPS, (b) EOS DI-II. The mass $M$ is shown in units of $M_{\odot}$, the radius $R_{e}$ in $\mathrm{km}$.

\section{A. Neutron star models and ISCO ranges}

Let us start by briefly recalling the mass-radius relation of the neutron star models studied in [45]. The physically relevant domain is delimited by several sequences of neutron star solutions, which comprise (i) the static neutron stars up to the maximum mass, (ii) the set of neutron stars along the secular instability line, formed by the set of neutron stars with maximum mass at fixed angular momentum, and (iii) the set of neutron stars rotating at the Kepler limit. In the Kepler limit the angular velocity $\Omega_{K}$ is reached, which corresponds to the angular velocity of a particle in geodesic motion at the equatorial surface of the star.

The mass-radius relation for the above limiting sequences of neutron star models for the two EOSs FPS and EOS DI-II and three values of the GB coupling constant, $\alpha=0,1$ and 2 , is exhibited in Fig. 1. The value $\alpha=0$ corresponds to the case of GR, which features the largest domain of existence of physically relevant models. With increasing GB coupling constant the domain and the associated maximal mass decrease.

Evidently, ISCOs should reside outside the neutron stars. Thus the neutron star surface represents an inner boundary for the existence of ISCOs. ISCOs then exist for neutron star models only above a minimum mass. In GR the dependence of this minimum mass on the angular velocity of the stars has been studied for various EOS 62, 63]. Here we address this minimum mass in dEGB theory.

For that purpose we exhibit in Fig. 2 again the physically relevant domain in mass-radius diagrams (left column), but now also in mass-energy density diagrams (right column), for the EOSs FPS ( $\alpha=1$ : upper panels, $\alpha=2$ : middle panels) and EOS DI-II ( $\alpha=2$ : lower panels). The colored lines inside these domains indicate the presence of ISCOs for sets of neutron star models with fixed rotational velocity $\Omega$ and for coupling parameter $\beta=0$. As long as these colored lines are solid, the numerical evidence for their existence is solid. However, when the numerical accuracy for the effective potential is no longer very high, it is hard to decide, whether an ISCOs still exists. The numerical evidence then becomes less clear, and we have denoted this uncertain range with dashed lines.

As an example for such a less clear cut case, we exhibit in Fig. 3 (left) the effective potential $V_{+}$for a rapidly rotating neutron star model with $\Omega=0.035$ for the EOSs FPS, GB coupling $\alpha=2$, matter coupling $\beta=0.0$, and ISCO angular momentum $L=L_{\mathrm{ISCO}}$. The dots indicate the mesh points where the solution is computed. The solid curve corresponds to the interpolated values. We note that the situation is similar for $\beta=0$ and $\beta=0.5$. For comparison Fig. 3 (right) shows the effective potential $V_{+}$for $L=L_{\mathrm{ISCO}}$ in case the ISCO is located at some larged distance from the star boundary, where the numerical accuracy is much better.

As a general trend we extract from theses calculations, that the higher the angular velocity of the star, the higher the minimum mass needed for ISCOs to exist. This trend seen in dEGB models is following the one seen in GR models. But just as the overall maximum mass decreases with increasing dEGB coupling $\alpha$, the minimum mass decreases with increasing $\alpha$, as well. 

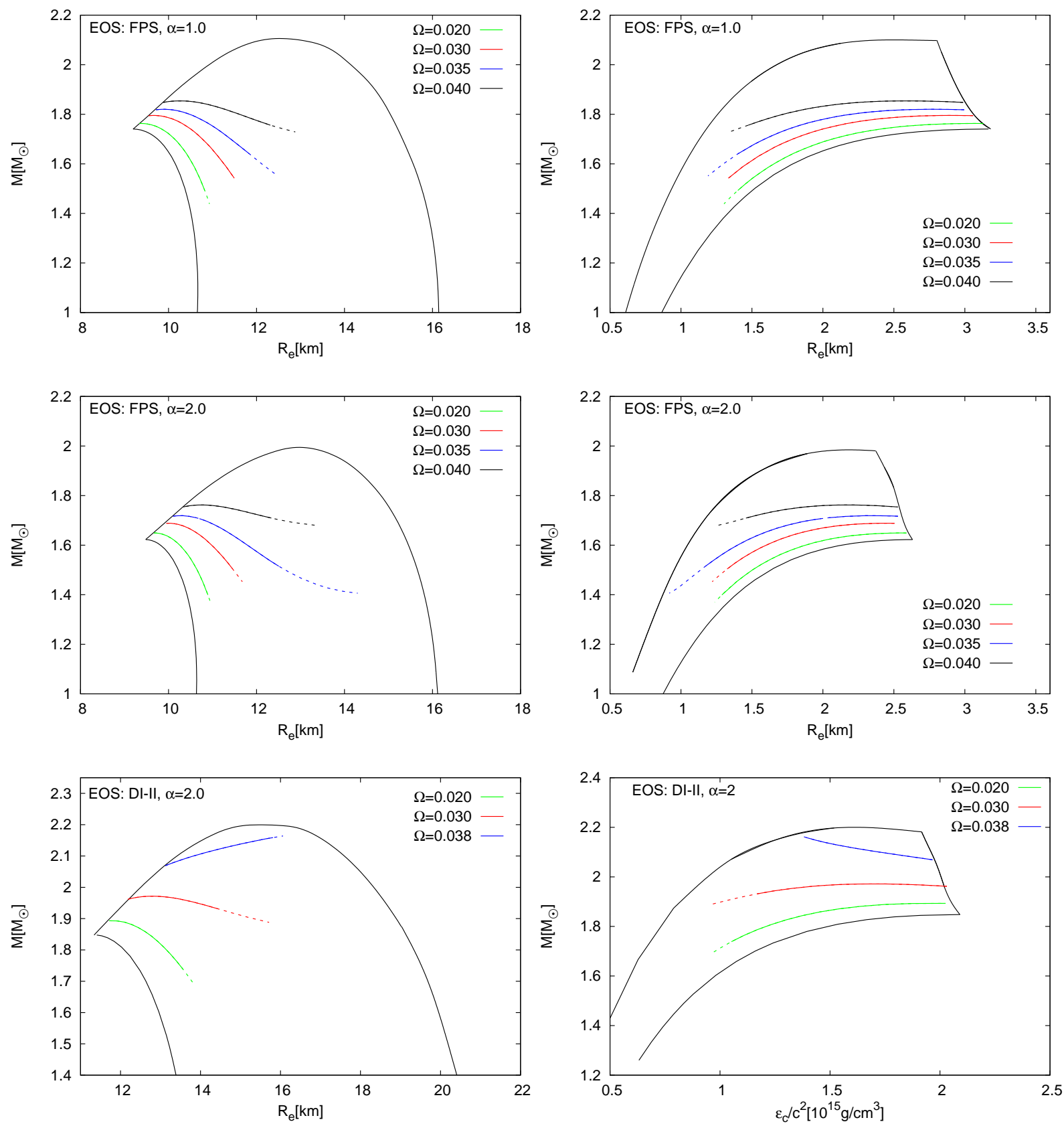

FIG. 2: Range of the existence of ISCOs for coupling $\beta=0$ for fixed angular velocities $\Omega$ of neutron star models for the EOSs FPS $(\alpha=1,2)$ and EOS DI-II $(\alpha=2)$. Solid lines indicate solid numerical evidence, dashed lines uncertain (see text) numerical evidence. The range is indicated in the corresponding mass-radius diagram in the left set of figures and in the mass-energy density diagram in the right set of figures. 

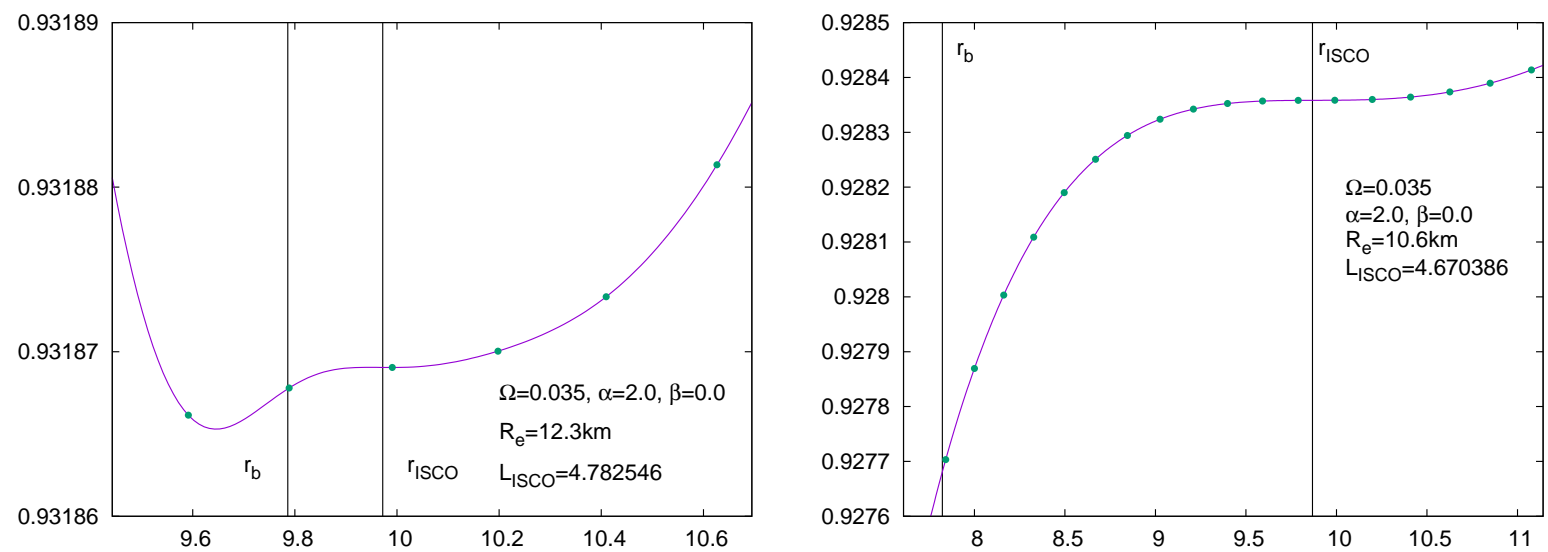

FIG. 3: Effective potential $V_{+}$for angular velocity $\Omega=0.035$ of the neutron star model, EOSs FPS, GB coupling constant $\alpha=2$, matter coupling $\beta=0$, and equatorial radius of the star $R_{\mathrm{e}}=12.3 \mathrm{~km}$ (left), and $R_{\mathrm{e}}=10.6 \mathrm{~km}$ (right). Indicated are the radial surface coordinate of the neutron star $r_{b}$ and the coordinate location of the (tentative) ISCO $r_{\text {ISCO. }}$

\section{B. ISCO radii and frequencies}

The ISCO has been studied in GR for rotating neutron stars subject to many diffent EOS (see e.g., [5263]). Let us now consider the ISCOs in dEGB theory in some detail and their dependence on the GB coupling constant $\alpha$, employing the above sets of neutron star models [45].

In particular, let us consider sets for fixed EOS and fixed $\alpha$, and either i) fixed scaled angular momentum $j=J / M^{2}$ of the star, or ii) fixed angular velocity $\Omega$ of the star. Let us note, that $\Omega=0.01$ corresponds to a rotation frequency of $323 \mathrm{~Hz}$, while the fastest spinning known neutron star has a frequency of $716 \mathrm{~Hz}$ [70], i.e., $\Omega \approx 0.022$.
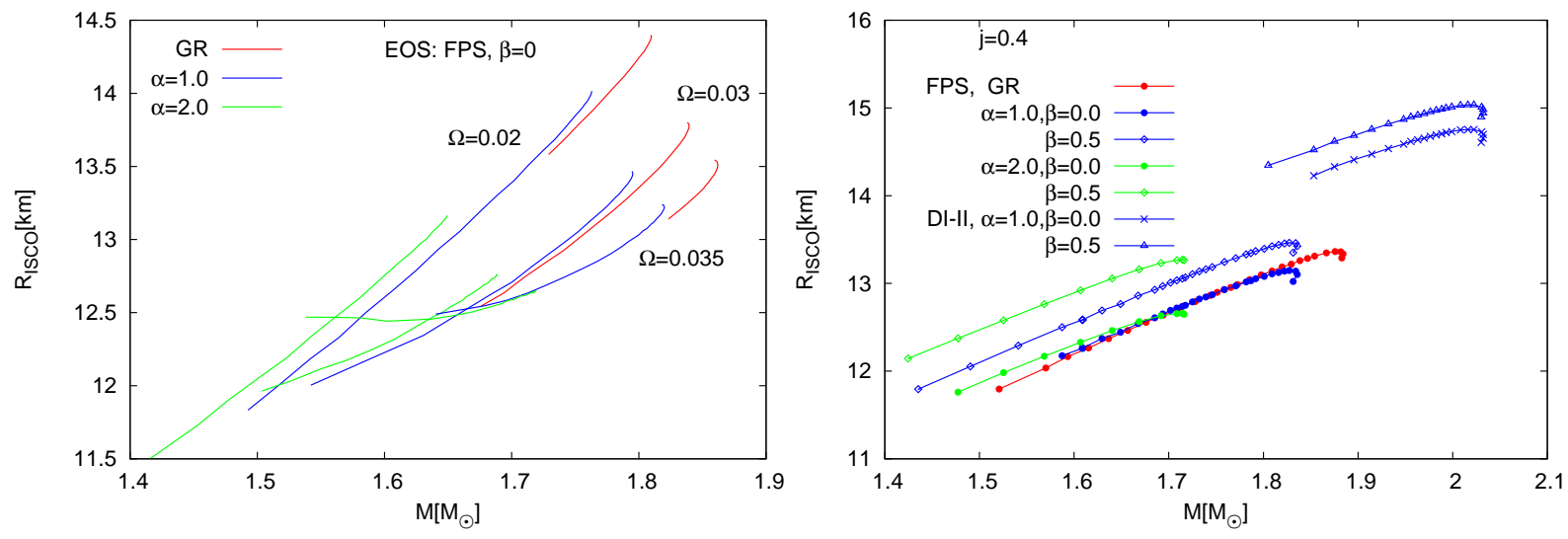

FIG. 4: ISCO radius $R_{\mathrm{ISCO}}$ in km versus the neutron star mass $M$ in solar masses $M_{\odot}$. Left: Fixed values of the angular velocity $\Omega$ of the star, $\Omega=0.02,0.03$ and 0.035 , matter coupling $\beta=0$, EOS FPS, for GR (red), dEGB with $\alpha=1.0$ (blue) and dEGB with $\alpha=2.0$ (green). Right: fixed value of the scaled angular momentum $j=0.4$ of the star, matter coupling $\beta=0$ (dots) and $\beta=0.5$ (diamonds), EOS FPS, for GR (red), dEGB with $\alpha=1.0$ (blue) and dEGB with $\alpha=2.0$ (green), as well as EOS DI-II, matter coupling $\beta=0$ (crosses) and $\beta=0.5$ (triangles) for dEGB with $\alpha=1.0$ (blue).

In Fig 4 we exhibit the radius of the ISCO $R_{\mathrm{ISCO}}$ in $\mathrm{km}$ versus the mass of the neutron star $M$ in units of the solar mass $M_{\odot}$ for several families of rapidly rotation neutron star models. Fig 4 (left) exhibits results for the EOS FPS, where the GB coupling $\alpha$ is fixed to zero, i.e., reproducing GR, to $\alpha=1$ and $\alpha=2$. Also fixed are the value of the matter coupling $\beta=0$, and the angular velocity of the stars, which assumes values $\Omega=0.02$, 0.03 and 0.035 . As seen in the figure, the different values of $\Omega$ lead to shifts towards smaller ISCO radii for 
given GB values. Likewise, an increase in the GB coupling constant leads to smaller ISCO radii for a given $\Omega$.

Fig 4 (right) on the other hand, considers families of neutron star models for a fixed value of the scaled angular momentum $j=0.4$. For the EOS FPS the GB coupling $\alpha$ is fixed to zero, i.e., reproducing GR, to $\alpha=1$ and $\alpha=2$, while the matter coupling assumes the values $\beta=0$ and $\beta=0.5$. In addition, the families for $\alpha=1$ and $\beta=0$ and $\beta=0.5$ are shown for the EOS DI-II. The figure shows, that the increase of $\beta$ leads to an increase of the ISCO radii, that rises strongly with the increase of $\alpha$. Moreover, the figure demonstrates the strong dependence of the ISCO radius on the EOS.
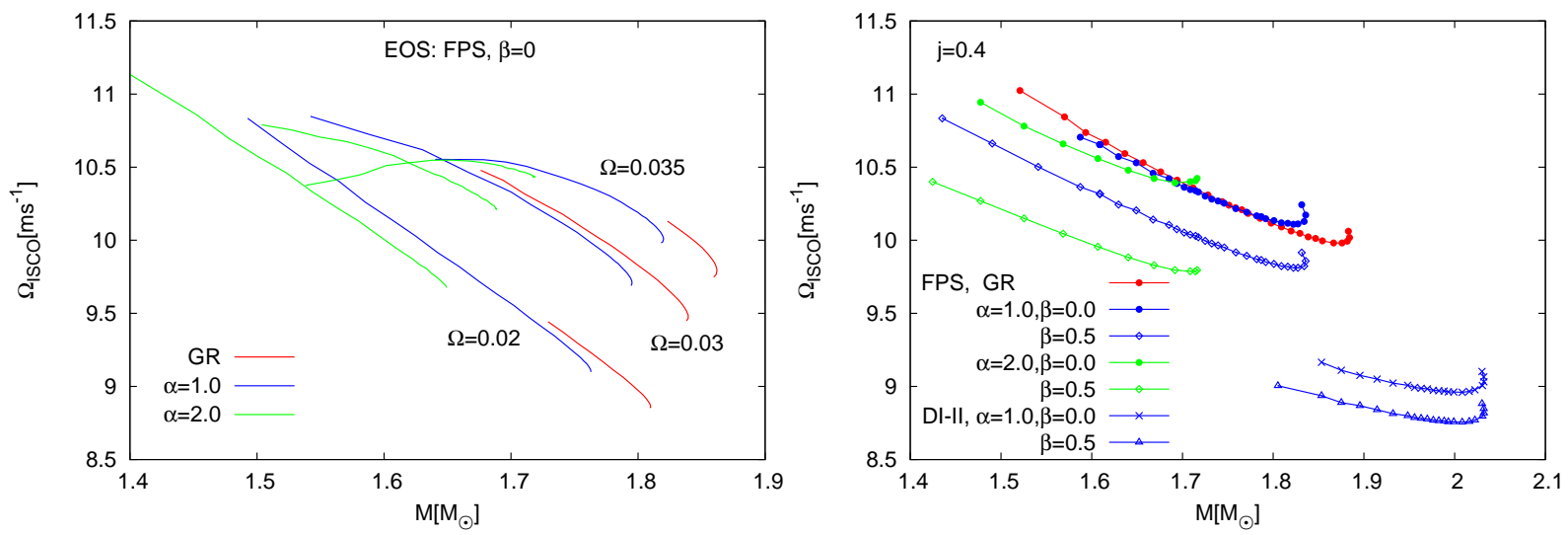

FIG. 5: ISCO angular velocity $\Omega_{\mathrm{ISCO}}$ in $\mathrm{ms}^{-1}$ versus the neutron star mass $M$ in solar masses $M_{\odot}$. Left: Fixed values of the angular velocity $\Omega$ of the star, $\Omega=0.02,0.03$ and 0.035 , matter coupling $\beta=0$, EOS FPS, for GR (red), dEGB with $\alpha=1.0$ (blue) and dEGB with $\alpha=2.0$ (green). Right: fixed value of the scaled angular momentum $j=0.4$ of the star, matter coupling $\beta=0$ (dots) and $\beta=0.5$ (diamonds), EOS FPS, for GR (red), dEGB with $\alpha=1.0$ (blue) and dEGB with $\alpha=2.0$ (green), as well as EOS DI-II, for dEGB, matter coupling $\beta=0$ (crosses) and $\beta=0.5$ (triangles) with $\alpha=1.0$ (blue).

In Fig. [5 we exhibit the ISCO angular velocity $\Omega_{\mathrm{ISCO}}$ in $\mathrm{ms}^{-1}$ versus the mass of the neutron star $M$ in units of the solar mass $M_{\odot}$ for the same families of rapidly rotating neutron star models as in Fig. 4. As seen in Fig 5 (left), the different values of the angular velocity $\Omega$ of the star now lead to shifts towards smaller ISCO angular velocities for given GB values, while an increase in the GB coupling constant leads to smaller ISCO angular velocities for a given angular velocity $\Omega$ of the star. Fig. 5 (right) shows, that the increase of the matter coupling $\beta$ now leads to a decrease of the ISCO angular velocities, that grows with increasing $\alpha$. In addition, the figure demonstrates the strong dependence of the ISCO angular velocity on the EOS.

In Fig. 6 we show the analogous sets of curves for the ISCO angular momentum $L_{\text {ISCO }}$ in $\mathrm{km}^{2} \mathrm{~s}^{-1}$ versus the neutron star mass. Here the dependencies on the parameters and EOS are also present but weaker than in the previous two cases, making the ISCO angular momentum $L_{\mathrm{ISCO}}$ a good new candidate when looking for universal relations.

\section{Universal relations}

Universal relations represent a unique means to learn about the EOS and the theory of gravity [26, 27]. In these universal relations one typically considers appropriately scaled physical properties, representing dimensionless quantities, and studies their various relations. For the scaling itself then the mass, radius or frequency of the neutron stars are invoked with appropriate powers.

When the $I$-Love- $Q$ relations were generalized for rapidly rotating neutron stars, it was realized, that the $I$ Love- $Q$ relations did not yield single (best fit) curves but full surfaces [30 32]. Here the additional dimensionless physical quantity could be represented by the scaled angular momentum $j$ or the scaled frequency in the form $\Omega M$ or $\Omega R$, but in each case corresponding to an additional dependence on the rotation of the neutron star.

Recently an analysis of ISCO properties by Luk and Lin [63] has led to a set of new universal relations in GR. There it was observed, that when $R_{\mathrm{ISCO}} \Omega$, i.e., the ISCO radius scaled by the star angular velocity, is considered as a function of $M \Omega$, i.e., the mass of the star scaled by its angular velocity, the data points from 12 different EOS fall more of less on a single curve, with deviations from this fitted universal curve on the order 

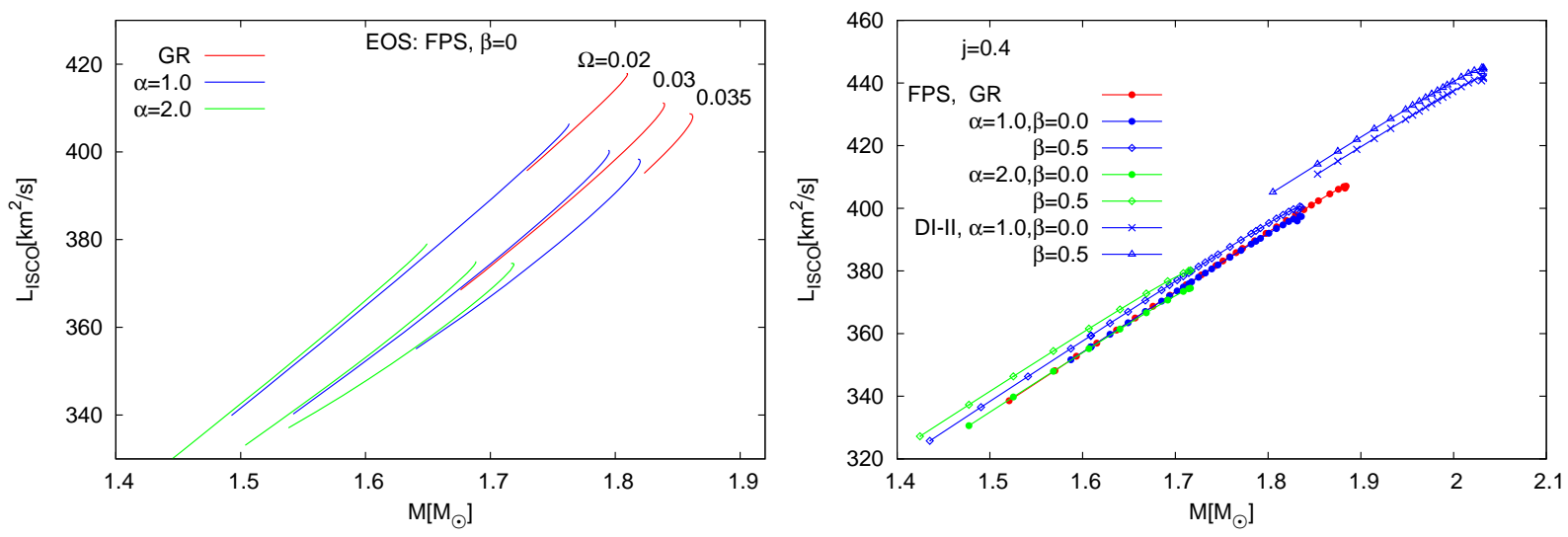

FIG. 6: ISCO angular momentum $L_{\mathrm{ISCO}}$ in $\mathrm{km}^{2} \mathrm{~s}^{-1}$ versus the neutron star mass $M$ in solar masses $M_{\odot}$. Left: Fixed values of the angular velocity $\Omega$ of the star, $\Omega=0.02,0.03$ and 0.04 , matter coupling $\beta=0$, EOS FPS, for GR (red), dEGB with $\alpha=1.0$ (blue) and dEGB with $\alpha=2.0$ (green). Right: fixed value of the scaled angular momentum $j=0.4$ of the star, matter coupling $\beta=0$ (dots) and $\beta=0.5$ (diamonds), EOS FPS, for GR (blue), dEGB with $\alpha=1.0$ (red) and dEGB with $\alpha=2.0$ (green), as well as EOS DI-II, matter coupling $\beta=0$ (crosses) and $\beta=0.5$ (triangles) for dEGB with $\alpha=1.0$ (blue).

of up to $6 \%$. Similarly, when instead $\Omega / \Omega_{\mathrm{ISCO}}$, i.e., the scaled ISCO angular velocity, is considered, the data points from the same set of EOS yield a fitted universal curve with deviations of the same order.

These universal relations of Luk and Lin [63] (in terms of the frequency $f=\Omega / 2 \pi$ ) correspond to

$$
y=a_{1} x+a_{2} x^{2}+a_{3} x^{3}+a_{4} x^{4},
$$

where for $y=R_{\mathrm{ISCO}} f$ and $x=M f$, the fitting parameters are $a_{1}=8.809, a_{2}=-9.166 \times 10^{-4}, a_{3}=$ $8.787 \times 10^{-8}, a_{4}=-6.019 \times 10^{-12}$, while for $y=f / f_{\text {ISCO }}$ the fitting parameters are given by $b_{1}=4.497 \times$ $10^{-4}, b_{2}=-6.130 \times 10^{-8}, b_{3}=4.527 \times 10^{-12}, b_{4}=-1.446 \times 10^{-16}$. For these universal curves the relative deviations $(\hat{y}-y) / y$ of the data points $\hat{y}$ are then on the few percent level for a very large range of stars, with $0 \leq M f \leq 5000 M_{\odot} \mathrm{Hz}$.

When comparing our GR data to these two fits, we find complete agreement, with the two universal relations of [63] being satisfied within $1.5 \%$, respectively $2.5 \%$. However, here our goal is to see the effects of the dEGB action and the associated coupling constants $\alpha$ and $\beta$. Let us therefore inspect what happens, as we consider the data of Fig. 4 (right) and Fig. 5 (right), appropriately scaled to match the above universal relations, as exhibited in Fig. 7 .

The figures show that universality is very well satisfied for GR and dEGB with $\alpha=1$ and $\beta=0$. Both satisfy more or less the same fits. For $\beta=0.5$, however, universality is slightly less well satisfied. In particular, we notice that new fits should be made for dEGB with $\alpha=1$ and $\beta=0.5$, since the deviations from GR are getting larger, exceeding even slightly the $\alpha=2, \beta=0$ deviations. For $\alpha=2$ and $\beta=0.5$ the deviations from GR become even larger. Thus observations of such large discrepancies between the scaled ISCO radii or angular velocities might allow to conclude, that a generalized theory of gravity might be needed.

We illustrate the full set of data and their deviations from their respective fits in Fig. 9. Together with the data points shown in Fig. 9 for the scaled ISCO radius $R_{\mathrm{ISCO}} \Omega$ (upper left) and the scaled ISCO frequency $f / f_{\text {ISCO }}$ (lower left) we exhibit the respective curves for the best fit for GR [63], for dEGB with $\alpha=1, \beta=0.5$, and for dEGB with $\alpha=2, \beta=0$. The deviations from the corresponding best fits for the scaled ISCO radius $R_{\mathrm{ISCO}} \Omega$ (upper left) and the scaled ISCO frequency $f / f_{\mathrm{ISCO}}$ (lower left) are also exhibited in the figure. While the universality remains good for dEGB with $\alpha=1$ and $\beta=0$ (deviations of 4\%), it clearly deteriorates for dEGB with $\alpha=1, \beta=0.5$ (deviations of $8 \%$ ), $\alpha=2, \beta=0$ (deviations of $8 \%$ ), and becomes broken for dEGB with $\alpha=2, \beta=0.5$, where deviations reach $25 \%$.

Thus the full universality seen in these relations for GR and surviving to dEGB with $\alpha=1$ and $\beta=0$ is giving way to a restricted universality, where an additional dependence on another dimensionless quantity, such as $j$, is needed to describe the data properly, i.e., one should instead consider a surface for extracting universal relations. For such an analysis our data are, however, insuffcient. We note, that a large part of our data are based on constant values of the dimensionful quantity $\Omega$. We also note, that such a structure would then be 

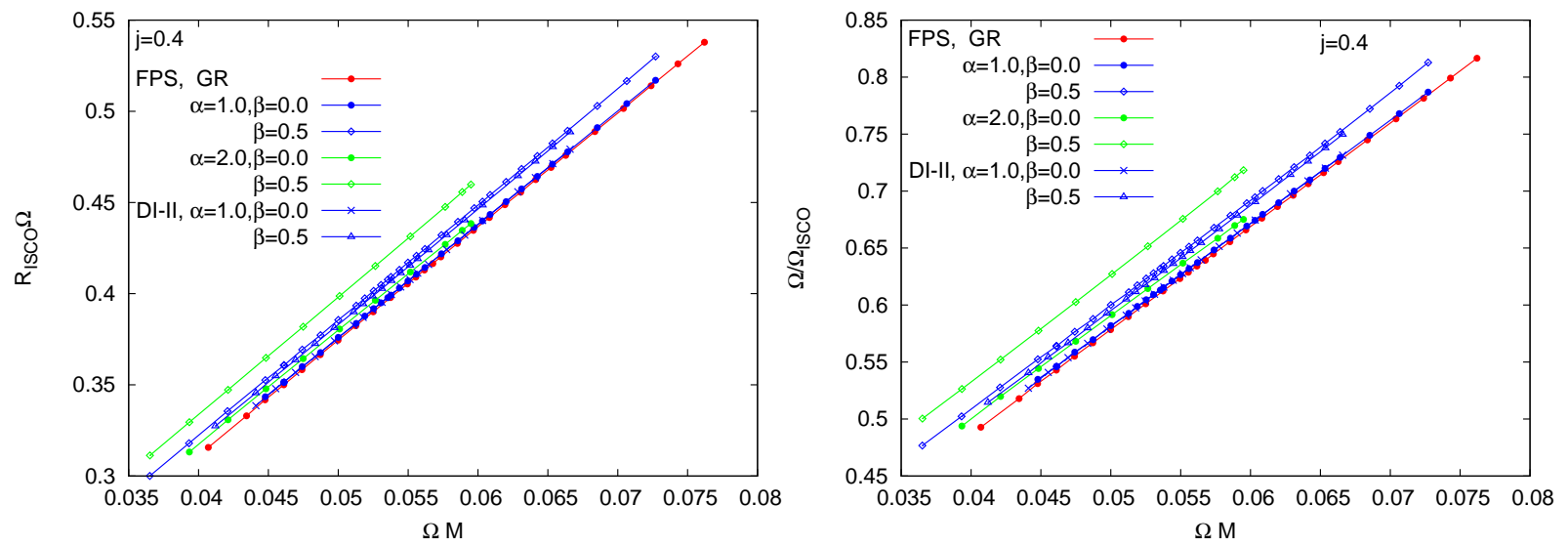

FIG. 7: Scaled ISCO radius $R_{\mathrm{ISCO}} \Omega$ (left) and scaled ISCO angular velocity $\Omega / \Omega_{\mathrm{ISCO}}$ (right) versus the scaled neutron star mass $M \Omega$. Fixed value of the scaled angular momentum $j=0.4$ of the star, matter coupling $\beta=0$ (dots) and $\beta=0.5$ (diamonds), EOS FPS, for GR (red), dEGB with $\alpha=1.0$ (blue) and dEGB with $\alpha=2.0$ (green), as well as EOS DI-II, for dEGB with $\alpha=1.0, \beta=0$ (crosses), and $\beta=0.5$ (triangles).
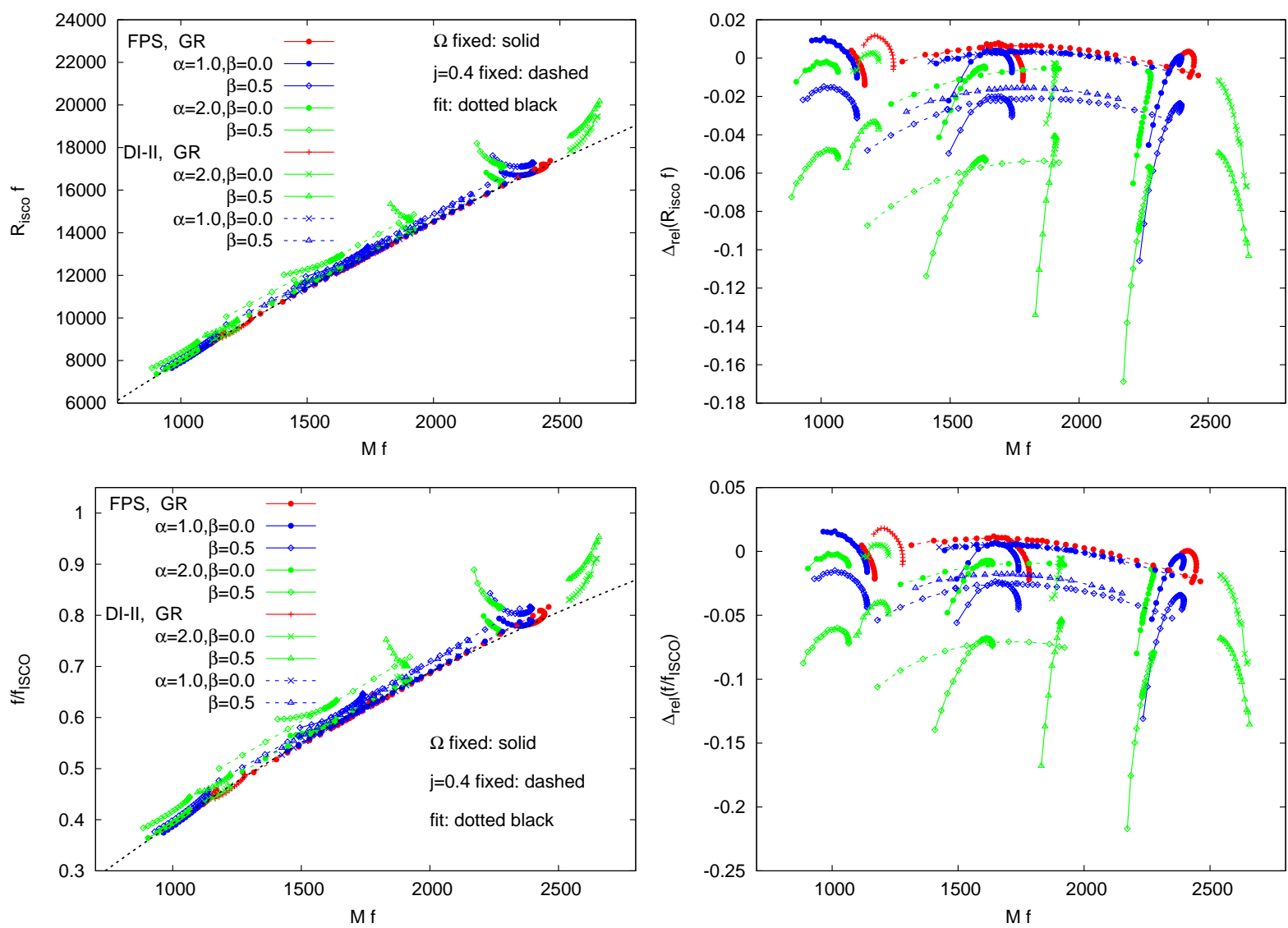

FIG. 8: Scaled ISCO radius $R_{\mathrm{ISCO}} f$ (upper left) and scaled ISCO frequency $f / f_{\mathrm{ISCO}}$ (lower left) versus the scaled neutron star mass $M f$, for GR (red), dEGB with $\alpha=1.0$ (blue) and dEGB with $\alpha=2.0$ (green), with EOS FPS, matter coupling $\beta=0$ (dots) and $\beta=0.5$ (diamonds), as well as EOS DI-II, for $\beta=0$ (crosses), and $\beta=0.5$ (triangles). Also shown are the relative deviations from the GR fits for the scaled ISCO radius (upper right) and the scaled ISCO frequency (lower right).

rather similar to the structure of the $I$-Love- $Q$ relations studied previously [30 32$]$. 

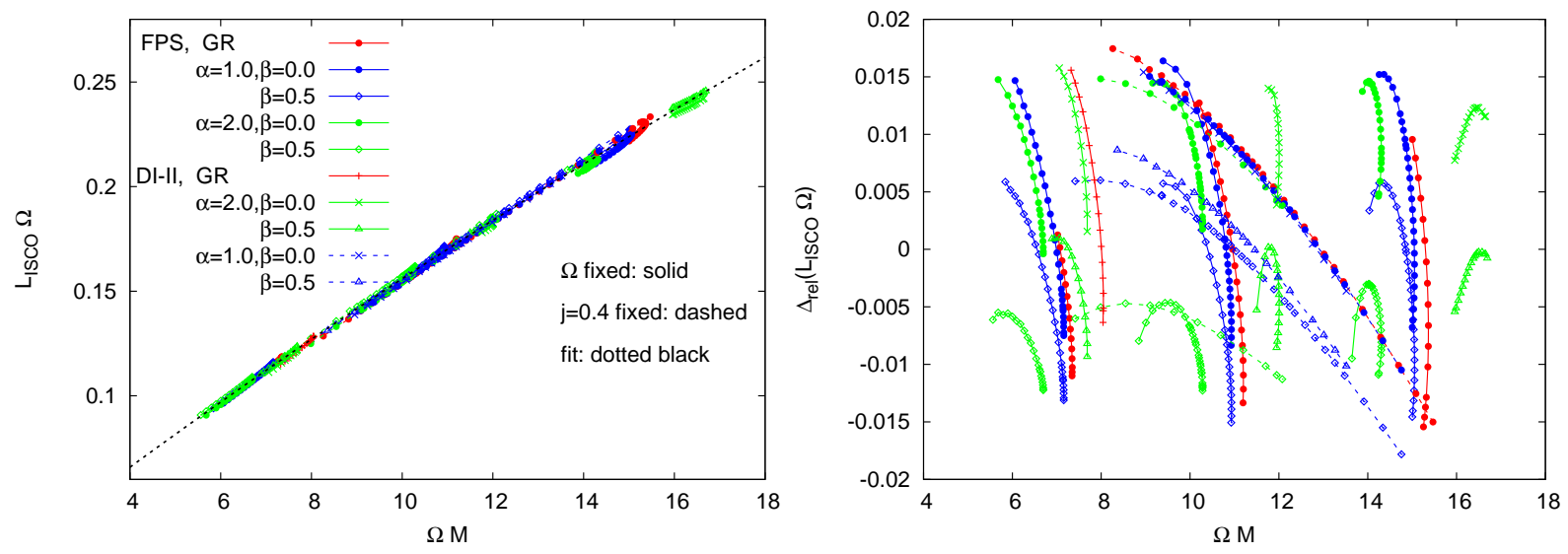

FIG. 9: Scaled ISCO angular momentum $L_{\mathrm{ISCO}} \Omega$ versus the scaled neutron star mass $M \Omega$ (left) and deviations from the fit (right). The data consist of both EOS, GR and dEGB with $\alpha=1,2, \beta=0,0.5$.

Considering the above results for the universal relations for $R_{\mathrm{ISCO}} f$ and $f / f_{\mathrm{ISCO}}$, it is all the more surprising that there exists a full universal relation for another ISCO property, namely for the scaled ISCO angular momentum $L_{\mathrm{ISCO}} \Omega$. This new universal relation is shown in Fig. 9, where all of the properly scaled data is superimposed. Clearly the data for both EOS, for GR, for dEGB with $\alpha=1$ and 2 , as well as $\beta=0$ and 0.5 all can be fitted by a single curve, and the deviations from this fit do not exceed $3 \%$. Let us recall here for comparison Fig. 6, which shows the unscaled quantities with their various dependencies. Thus the scaling removes all of these dependencies, resulting in a single universal curve. The universal relation reads

$$
L \Omega_{\mathrm{ISCO}}=c_{1} M \Omega+c_{2}(M \Omega)^{2}+c_{3}(M \Omega)^{3}
$$

with $c_{1}=1.707 \times 10^{-2}, c_{2}=-1.567 \times 10^{-4}, c_{3}=9.147 \times 10^{-7}$.
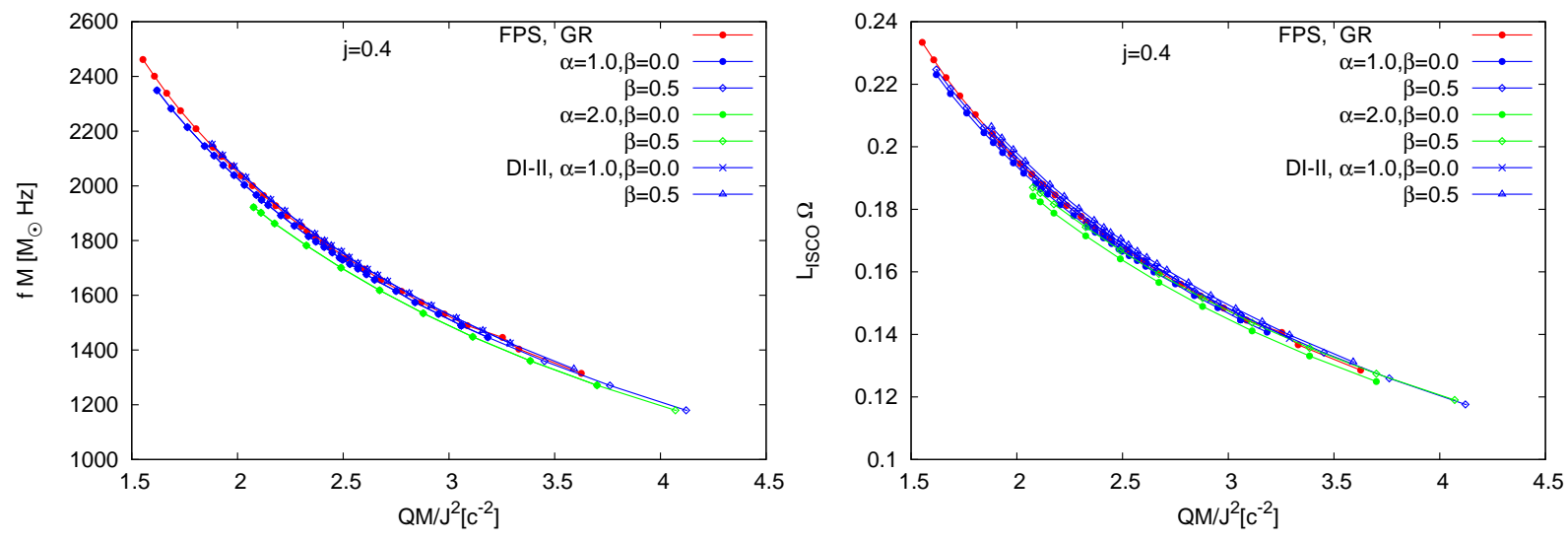

FIG. 10: Scaled neutron star mass $M f$ (left) and scaled ISCO angular momentum $L_{\mathrm{ISCO}} \Omega$ (right) versus the scaled quadrupole moment $Q M / J^{2}$ for a fixed value of the scaled angular momentum $j=0.4$ of the star, matter coupling $\beta=0$ (dots) and $\beta=0.5$ (diamonds), EOS FPS, for GR (red), dEGB with $\alpha=1.0$ (blue) and dEGB with $\alpha=2.0$ (green), as well as EOS DI-II, for dEGB with $\alpha=1.0, \beta=0$ (crosses), and $\beta=0.5$ (triangles).

Let us finally connect to previous universal relations [26, 27], and, in particular, to the universal $I$ - $Q$ relation in dEGB theory [44, 45]. This relation is known to depend on the dimensionless angular momentum $j$, and thus corresponds to a universal surface. Let us now consider a particular curve on this surface given by a fixed value of $j$. Then there is almost no dependence on the EOS and the parameters $\alpha$ and $\beta$ [44, 45]. If we consider instead the scaled mass $M \Omega$ versus the scaled quadrupole moment $Q M / J^{2}$, then this high degeneracy with respect to $\alpha$ and $\beta$ is slightly broken, as seen in Fig. 10 (left).

Not surprisingly, this slight breaking of the degeneracy is also visible, when the scaled ISCO angular momentum $L \Omega$ is considered versus the scaled quadrupole moment $Q M / J^{2}$, as shown in Fig 10 (right). We note, 
that for the dependence of the scaled ISCO radius $R_{\mathrm{ISCO}} \Omega$ or the scaled ISCO frequency $\Omega_{c} / \Omega$ on the scaled quadrupole moment $Q M / J^{2}$, the breaking of the degeneracy with $\alpha$ and $\beta$ increases as compared to $L \Omega$. In all cases, universal surfaces are expected to result, when data with different values of $j$ are included.
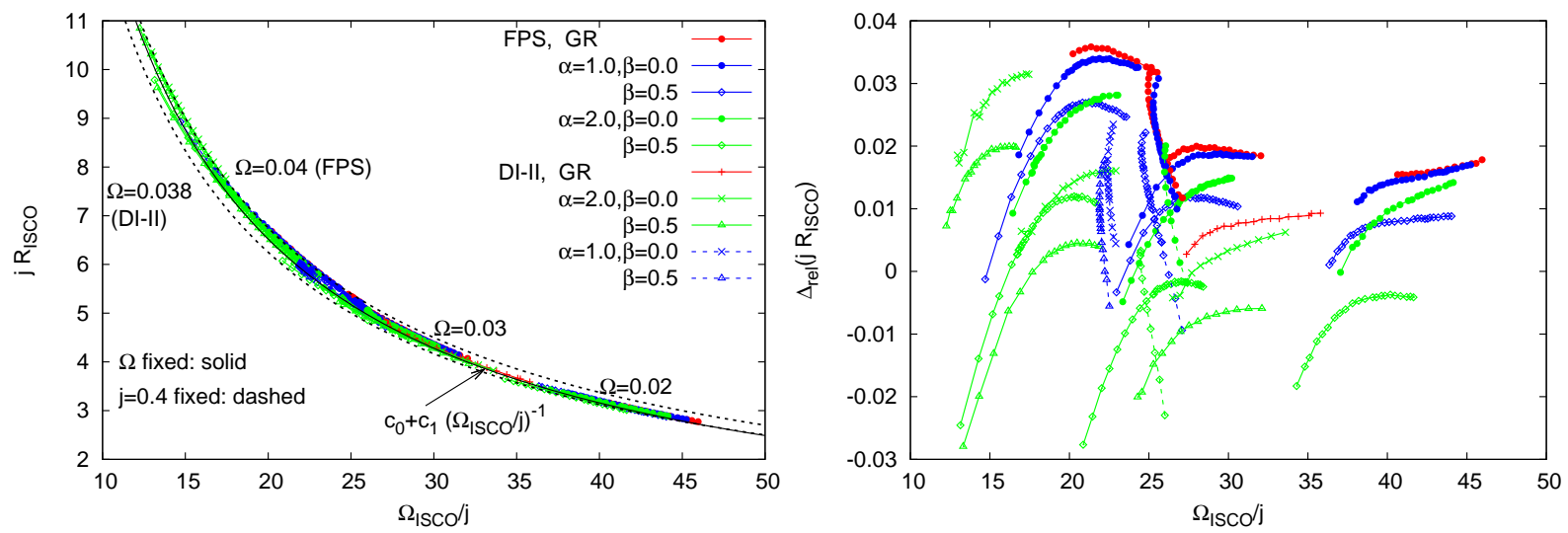

FIG. 11: Scaled ISCO radius $R_{\mathrm{ISCO}} j$ versus the scaled ISCO angular velocity $\Omega_{\mathrm{ISCO}} / j$ (left) and deviations from the fitted curve (right). The data consist of both EOS, GB and dEGB with $\alpha=1,2, \beta=0,0.5$.

As a last point of interest let us mention, that we have also observed a relation, that looks like a full universal relation, since a single fit covers all the data within 3\% accuracy, thus including the variations of $\alpha$ and $\beta$,

$$
R_{\mathrm{ISCO}} j=c_{0}+c_{1}\left(\Omega_{\mathrm{ISCO}} / j\right)^{-1},
$$

with $c_{0}=-0.2$ and $c_{1}=134.4$. However, this relation involves dimensionful quantities, the ISCO radius $R_{\mathrm{ISCO}}$ and ISCO angular velocity $\Omega_{\mathrm{c}}$, scaled by the dimensionless angular momentum $j$.

\section{CONCLUSIONS}

Since neutron stars are highly compact objects, they represent - in principle - a means to extract a lot of information about GR or generalized theories of gravity. However, there is a price to pay, since the properties of neutron stars are highly dependent on the EOS. Here universal relations represent an excellent means to eliminate this EOS dependence to a large extent.

While universal relations for properties of the neutron stars themselves have been studied in much detail before, a set of universal relations for the ISCOs of neutron stars in GR has only recently been announced 63]. These universal relations concern the dependence of the scaled ISCO radius $R_{\mathrm{ISCO}} \Omega$ and the scaled ISCO frequency $\Omega_{\mathrm{c}} / \Omega$ on the scaled neutron star mass $M \Omega$. Here we have added a further such relation, namely the dependence of the scaled ISCO angular momentum $L_{\mathrm{ISCO}} \Omega$ on the scaled mass $M \Omega$.

However, our main goal has been to gain some understanding on how these universal relations are affected, when a generalized theory of gravity is employed instead of GR. Therefore we have analyzed our neutron star data obtained in the string theory motivated dEGB gravity, with GB coupling constants $\alpha=1$ and 2 , allowing at the same time for varying values of the matter coupling constant $\beta=0$ and $\beta=0.5$, with the latter value corresponding to the value suggested by string theory.

Inspection of the GR universal relations $R_{\mathrm{ISCO}} \Omega$ versus $M \Omega$ and $\Omega / \Omega_{\mathrm{ISCO}}$ versus $M \Omega$ for dEGB has shown, that a small dependence on the GB coupling constant arises, as well as a larger dependence on the matter coupling constant $\beta$. Moreover, we conclude, that the single degenerate curve should in principle be replaced by a surface, where the scaled angular momentum $j$ could represent the additional axis.

Interestingly, the new GR universal relation $L_{\mathrm{ISCO}} \Omega$ versus $M \Omega$ remains basically untouched, as the GB coupling constant $\alpha$ and the matter coupling constant $\beta$ are varied. Indeed, for all available data the relative deviation from the GR fit does not exceed $3 \%$. Consequently this new universal relation does not discriminate between GR and dEGB theory, whereas the two previously found relations do, when $\alpha$ or $\beta$ is sufficiently large.

There has also been previous work concerning relations between ISCO properties and the multipole moments of neutron stars [53, 63, 71]. In particular, expansions have been considered, relating the ISCO radius and 
the ISCO frequency to dimensionless parameters like the scaled angular momentum $j$, the scaled quadrupole moment and the scaled spin octupole moment. Here we have considered the dependence of $L_{\mathrm{ISCO}} \Omega, R_{\mathrm{ISCO}} \Omega$ and $\Omega / \Omega_{\mathrm{ISCO}}$ on the scaled quadrupole moment $Q M / J^{2}$. In all cases, we observe for fixed $j$ a small dependence on the dEGB parameters, and we expect universal surfaces when $j$ is varied as well.

As a final curious finding we note, that we have also encountered a relation for dimensionful quantities, that otherwise looks like a universal relation, valid both for GR and dEGB. Here we have considered $R_{\mathrm{ISCO}} j$ versus $j / \Omega_{\text {ISCO }}$. Thus the ISCO properties are dimensionful, while they are scaled with the dimensionless $j$. The deviations from the fitted curve are below $4 \%$ for this intriguing relation.

If observations would indicate sufficiently strong deviations from the GR universal relations, this would suggest the need for a more general theory of gravity, allowing for such deviations. We have seen, that dEGB theory can accomplish deviations for certain GR universal relations, while remaining in perfect agreement with certain other GR universal relations. Viewing dEGB theory as a particular case of Horndeski gravity [8] then suggests to extend the current set of investigations in order to address further cases of Horndeski gravity in the future.

Here one might, in particular, focus on candidate theories that are also in good agreement with gravitational waves tests at the cosmological level [72]. We note, that there are already various interesting results on geodesic motion and accretion processes of black holes in Horndeski theories [73, 74], while neutron stars have also been investigated in Horndeski theories with a focus on their global properties, their modes and the associated universal relations (see e.g. [75-78]).

Let us end with some comments concerning proposed applications. The ISCO may be related to QPOs of low mass x-ray binaries, where the QPOs are attributed to oscillations close to the edge of the accretion disk, that is given by the respective ISCO (see e.g., [66, 67]). When the frequency of the neutron star and QPO frequency associated with the ISCO are known, then the GR universal relation would allow a determination of the neutron star mass 63]. For dEGB gravity on the other hand, this simple relation would be lost, since with $\alpha$ and $\beta$ two unknown parameters enter the universal relations. If, however, one could find a way to observationally extract the ISCO angular momentum, then the neutron star mass could also be determined in dEGB gravity.

\section{Acknowledgements}

This research was partially supported by VIE-UIS, under grant number 2416, and COLCIENCIAS grant number 110277657744 (VIE-UIS 8863), Colombia. SM wants to thank the support from the Postdoctoral Fellowship Scheme provided by VIE-UIS. BK and JK gratefully acknowledge support by the DFG Research Training Group 1620 Models of Gravity and the COST Action CA16104.

[1] S. Capozziello and M. De Laurentis, Phys. Rept. 509, 167 (2011)

[2] C. M. Will, Living Rev. Rel. 17, 4 (2014)

[3] E. Berti et al., Class. Quant. Grav. 32, 243001 (2015)

[4] B. Zwiebach, Phys. Lett. 156B, 315 (1985).

[5] D. J. Gross and J. H. Sloan, Nucl. Phys. B 291, 41 (1987).

[6] R. R. Metsaev, A. A. Tseytlin, Nucl. Phys. B293 , 385 (1987).

[7] F. Moura and R. Schiappa, Class. Quant. Grav. 24, 361 (2007)

[8] T. Kobayashi, M. Yamaguchi and J. Yokoyama, Prog. Theor. Phys. 126 (2011) 511

[9] S. Mignemi and N. R. Stewart, Phys. Rev. D 47 (1993) 5259

[10] S. Mignemi, Phys. Rev. D 51 (1995) 934

[11] P. Kanti, N. E. Mavromatos, J. Rizos, K. Tamvakis and E. Winstanley, Phys. Rev. D 54 (1996) 5049

[12] T. Torii, H. Yajima and K. i. Maeda, Phys. Rev. D 55 (1997) 739

[13] S. O. Alexeev and M. V. Pomazanov, Phys. Rev. D 55 (1997) 2110

[14] Z. K. Guo, N. Ohta and T. Torii, Prog. Theor. Phys. 120 (2008) 581

[15] P. Pani and V. Cardoso, Phys. Rev. D 79 (2009) 084031

[16] P. Pani, C. F. B. Macedo, L. C. B. Crispino and V. Cardoso, Phys. Rev. D 84 (2011) 087501

[17] B. Kleihaus, J. Kunz and E. Radu, Phys. Rev. Lett. 106 (2011) 151104

[18] D. Ayzenberg and N. Yunes, Phys. Rev. D 90, 044066 (2014) Erratum: [Phys. Rev. D 91, no. 6, 069905 (2015)]

[19] A. Maselli, P. Pani, L. Gualtieri and V. Ferrari, Phys. Rev. D 92 (2015) 8, 083014 arXiv:1507.00680 [gr-qc]].

[20] B. Kleihaus, J. Kunz, S. Mojica and E. Radu, Phys. Rev. D 93, no. 4, 044047 (2016)

[21] J. M. Lattimer, Ann. Rev. Nucl. Part. Sci. 62, 485 (2012) 
[22] F. Özel and P. Freire, Ann. Rev. Astron. Astrophys. 54, 401 (2016).

[23] G. Baym, T. Hatsuda, T. Kojo, P. D. Powell, Y. Song and T. Takatsuka, Rept. Prog. Phys. 81, no. 5, 056902 (2018)

[24] P. Demorest, T. Pennucci, S. Ransom, M. Roberts and J. Hessels, Nature 467, 1081 (2010)

[25] J. Antoniadis et al., Science 340, 6131 (2013)

[26] K. Yagi and N. Yunes, Phys. Rept. 681, 1 (2017).

[27] D. D. Doneva and G. Pappas, arXiv:1709.08046 [gr-qc].

[28] K. Yagi and N. Yunes, Science 341, 365 (2013)

[29] K. Yagi and N. Yunes, Phys. Rev. D 88, 023009 (2013)

[30] D. D. Doneva, S. S. Yazadjiev, N. Stergioulas and K. D. Kokkotas, Astrophys. J. 781, L6 (2013)

[31] G. Pappas and T. A. Apostolatos, Phys. Rev. Lett. 112, 121101 (2014)

[32] S. Chakrabarti, T. Delsate, N. Gürlebeck and J. Steinhoff, Phys. Rev. Lett. 112, 201102 (2014)

[33] K. Yagi, K. Kyutoku, G. Pappas, N. Yunes and T. A. Apostolatos, Phys. Rev. D 89, 124013 (2014)

[34] K. Yagi, L. C. Stein, G. Pappas, N. Yunes and T. A. Apostolatos, Phys. Rev. D 90, 063010 (2014)

[35] J. B. Hartle, Astrophys. J. 150, 1005 (1967).

[36] G. B. Cook, S. L. Shapiro and S. A. Teukolsky, Astrophys. J. 422, 227 (1994).

[37] G. B. Cook, S. L. Shapiro and S. A. Teukolsky, Astrophys. J. 424, 823 (1994).

[38] N. Stergioulas, Living Rev. Rel. 6, 3 (2003)

[39] E. Gourgoulhon, arXiv:1003.5015 [gr-qc].

[40] J. L. Friedman and N. Stergioulas, "Rotating Relativistic Stars," Cambridge University Press, Cambridge 2013.

[41] F. Cipolletta, C. Cherubini, S. Filippi, J. A. Rueda and R. Ruffini, Phys. Rev. D 92, 023007 (2015)

[42] P. Pani, E. Berti, V. Cardoso and J. Read, Phys. Rev. D 84, 104035 (2011)

[43] P. Pani and E. Berti, Phys. Rev. D 90 (2014) 2, 024025

[44] B. Kleihaus, J. Kunz and S. Mojica, Phys. Rev. D 90, 061501 (2014)

[45] B. Kleihaus, J. Kunz, S. Mojica and M. Zagermann, Phys. Rev. D 93, no. 6, 064077 (2016)

[46] D. D. Doneva, S. S. Yazadjiev, K. V. Staykov and K. D. Kokkotas, Phys. Rev. D 90, 104021 (2014)

[47] J. Diaz-Alonso and J.M. Ibañez-Cabanell, Astrophys. J. 291, 308 (1985).

[48] C. P. Lorenz, D. G. Ravenhall and C. J. Pethick, Phys. Rev. Lett. 70, 379 (1993).

[49] P. Haensel and A. Y. Potekhin, Astron. Astrophys. 428, 191 (2004)

[50] B. Carter, Phys. Rev. 174 (1968) 1559.

[51] J. M. Bardeen, Astrophys. J. 161, 103 (1970).

[52] M. C. Miller, F. K. Lamb and G. B. Cook, Astrophys. J. 509, 793 (1998)

[53] M. Shibata and M. Sasaki, Phys. Rev. D 58, 104011 (1998)

[54] J. L. Zdunik, P. Haensel, D. Gondek-Rosinska and E. Gourgoulhon, Astron. Astrophys. 356, 612 (2000)

[55] D. Gondek-Rosinska, T. Bulik, W. Kluzniak, J. L. Zdunik and E. Gourgoulhon, ESA Spec. Publ. 459, 223 (2001)

[56] L. A. Pachon, J. A. Rueda and J. D. Sanabria-Gomez, Phys. Rev. D 73, 104038 (2006)

[57] S. Bhattacharyya, Mon. Not. Roy. Astron. Soc. 415, 3247 (2011)

[58] G. Pappas and T. A. Apostolatos, Mon. Not. Roy. Astron. Soc. 429, 3007 (2013).

[59] G. Pappas, Mon. Not. Roy. Astron. Soc. 422, 2581 (2012)

[60] D. Gondek-Rosinska, W. Kluzniak, N. Stergioulas and M. Wisniewicz, Phys. Rev. D 89, no. 10, 104001 (2014)

[61] G. Torok, M. Urbanec, K. Adamek and G. Urbancova, Astron. Astrophys. 564, L5 (2014)

[62] F. Cipolletta, C. Cherubini, S. Filippi, J. A. Rueda and R. Ruffini, Phys. Rev. D 96, no. 2, 024046 (2017)

[63] S. S. Luk and L. M. Lin, Astrophys. J. 861, no. 2, 141 (2018)

[64] D. D. Doneva, S. S. Yazadjiev, N. Stergioulas, K. D. Kokkotas and T. M. Athanasiadis, Phys. Rev. D 90, no. 4, $044004(2014)$

[65] K. V. Staykov, D. D. Doneva and S. S. Yazadjiev, Eur. Phys. J. C 75, no. 12, 607 (2015)

[66] van der Klis, M., Astronomische Nachrichten 326, 798 (2005).

[67] M. van Doesburgh, M. van der Klis and S. Morsink, Mon. Not. Roy. Astron. Soc. 479, no. 1, 426 (2018).

[68] K. Yagi, Phys. Rev. D 86 (2012) 081504

[69] B. Kleihaus and J. Kunz, Phys. Rev. Lett. 86, 3704 (2001)

[70] J. W. T. Hessels, S. M. Ransom, I. H. Stairs, P. C. C. Freire, V. M. Kaspi and F. Camilo, Science 311, 1901 (2006)

[71] G. Pappas, Mon. Not. Roy. Astron. Soc. 454, no. 4, 4066 (2015)

[72] M. Ishak, arXiv:1806.10122 [astro-ph.CO].

[73] D. A. Tretyakova, J. Exp. Theor. Phys. 125, no. 3, 403 (2017) [Zh. Eksp. Teor. Fiz. 152, no. 3, 475 (2017)].

[74] K. Salahshoor and K. Nozari, Eur. Phys. J. C 78, no. 6, 486 (2018)

[75] A. Cisterna, T. Delsate and M. Rinaldi, Phys. Rev. D 92, no. 4, 044050 (2015)

[76] A. Maselli, H. O. Silva, M. Minamitsuji and E. Berti, Phys. Rev. D 93, no. 12, 124056 (2016)

[77] J. L. Blázquez-Salcedo and K. Eickhoff, Phys. Rev. D 97, no. 10, 104002 (2018)

[78] J. L. Blzquez-Salcedo, Z. Altaha Motahar, D. D. Doneva, F. S. Khoo, J. Kunz, S. Mojica, K. V. Staykov and S. S. Yazadjiev, arXiv:1810.09432 [gr-qc]. 